\title{
Clinical Characteristics and Risk Factors for Pleural Effusion in Patients with Multiple Myeloma
}

This article was published in the following Dove Press journal:

International Journal of General Medicine

\author{
Yu Kang (D) \\ Zi-Liang Hou ${ }^{2}$ \\ Guang-Zhong Yang (1D ${ }^{3}$ \\ Xiao-Juan Wang' \\ Wen-Ming Chen ${ }^{3}$ \\ Huan-Zhong Shi ${ }^{4}$ \\ 'Department of Internal Medicine, Beijing \\ Chao-Yang Hospital, Capital Medical \\ University, Beijing, People's Republic of \\ China; ${ }^{2}$ Department of Respiratory and \\ Critical Care Medicine, Beijing Luhe \\ Hospital, Capital Medical University, \\ Beijing, People's Republic of China; \\ ${ }^{3}$ Department of Hematology, Beijing \\ Chao-Yang Hospital, Capital Medical \\ University, Beijing, People's Republic of \\ China; ${ }^{4}$ Department of Respiratory and \\ Critical Care Medicine, Beijing Institute \\ of Respiratory Medicine, Beijing Chao- \\ Yang Hospital, Capital Medical University, \\ Beijing, People's Republic of China
}

Correspondence: Huan-Zhong Shi;

Wen-Ming Chen

Email shihuanzhong@sina.com;

13910107759@163.com
Purpose: Pleural effusion (PE) is prevalent in "real-life" populations of multiple myeloma (MM), a common hematologic malignancy. Development of PE likely has prognostic implications. The aim of this study was to investigate the characteristics and identify risk factors for occurrence of PE in MM.

Patients and Methods: We reviewed electronic medical records of 907 patients diagnosed with MM.

Results: Incidence of PE in MM patients was $42.7 \%$. Small and bilateral PE in most cases. PE developed in all MM subtypes, the median time from diagnosis of multiple myeloma to pleural effusion was 6.8 months (range 0.8-33.6 months). Patients with PE showed worse survival than those without PE (unadjusted hazard ratio with $95 \%$ confidence interval: 2.249 [1.774-2.852]). No difference in survival was found between patients with small PE and those with moderate to large PE (unadjusted HR, 1.402; 95\% CI, 1.037-1.896). Plasma cell proportion (OR, 1.373; 95\% CI, 1.153-1.634; $\mathrm{P}=0.009)$ and amyloidosis $(\mathrm{OR}, 1.791 ; 95 \%$ $\mathrm{CI}, 1.408-2.279 ; \mathrm{P}=0.024)$ were risk factors for the occurrence of $\mathrm{PE}$ at the initial diagnosis of MM. Plasma cell proportion (OR, 1.853; 95\% CI, 1.451-2.368; $\mathrm{P}=0.038$ ), pneumonia (OR, 1.309; 95\% CI, 1.143-1.498; $\mathrm{P}=0.008)$ and heart failure $(\mathrm{OR}, 1.815 ; 95 \% \mathrm{CI}$, 1.387-2.374; $\mathrm{P}=0.031$ ) were risk factors for the occurrence of $\mathrm{PE}$ at relapse of MM.

Conclusion: The incidence of PE in MM patients is notable and PE can occur in all MM subtypes. PE indicates a poor prognosis, even small amounts of effusion. PE is a problem worthy of attention, especially in patients with high plasma cell proportion, amyloidosis or complicated with pneumonia and heart failure.

Keywords: pleural effusion, multiple myeloma, incidence, risk factors, overall survival

\section{Introduction}

Pleural effusion (PE) is a common clinical problem. It is estimated that about 1.5 million people suffer from PE in the United States. ${ }^{1}$ PE can be caused by a variety of reasons, including disease local to the pleura or systemic condition. ${ }^{2-4}$ Hematologic malignances are one of the causes of pleural effusion. Appearance of PE portends a poorer prognosis in cancer patients. ${ }^{5,6}$ It was reported that appearance of PE is associated with clinical response during hematologic malignances treatment., ${ }^{7,8}$ Evaluation of pleural effusion in patients with hematologic malignances is required.

Multiple myeloma (MM) is the second most frequent hematologic malignancy, and each year over 30,000 new cases are diagnosed in the USA. ${ }^{9,10}$ In Asian countries, the incidence of MM has increased rapidly during the past two decades, and MM now represents the third most common hematologic disease in South Korea. ${ }^{11,12}$ It is estimated that incidence of MM patients in China was 27,800 new cases each year 
and a total of 200000 cases in China. ${ }^{13}$ Multiple myeloma is a clonal B-cell malignancy characterized by proliferation of uncontrolled plasma cells in the bone marrow or extramedullary sites, leading to excessive production of immunoglobulins. Anemia, renal failure, hypercalcemia, and lytic bone lesions are the most commonly encountered manifestations of MM. ${ }^{14}$

In our center, $\mathrm{PE}$ is frequently diagnosed in patients with MM. Previous literature reported the PE frequency of $10.7 \%$ to $13.9 \%{ }^{15,16}$ However, according to our observations, the incidence of $\mathrm{PE}$ in MM patients is much higher. Hitherto, articles on $\mathrm{PE}$ in MM patients were mostly case reports, comprehensive studies of PE in MM patients have not been performed. There is a pressing need to better understand the clinical characteristics and risk factors for $\mathrm{PE}$ in patients with MM. Thus, here we aimed to investigate the incidence, distribution, and outcomes of $\mathrm{PE}$ in patients with $\mathrm{MM}$, and to investigate risk factors for PE in MM patients.

\section{Patients and Methods}

\section{Study Population and Data Collection}

This was a retrospective, single-center study. We identified a total of the 907 patients who were diagnosed with multiple myeloma were admitted to Beijing Chao-yang Hospital between January 01, 2000, and December 31, 2017. There were 46 patients without computed tomography (CT) of the chest, which were excluded from the study. Among the enrolled $861 \mathrm{MM}$ patients, 528 (61.3\%) were newly diagnosed, and 208 (24.2\%) were relapsed multiple myeloma (Figure 1).

The detailed medical history, clinical presentation, laboratory results, and imaging data from all patients were extracted from the electronic medical records. We studied various aspects of PE associated with MM. The study protocol was approved by the Institutional Review Board for Human Studies of Beijing Chaoyang Hospital, Beijing, China. The study was carried out in conformity to the Declaration of Helsinki. The review board exempted the acquisition of informed consent because this was a retrospective study. Patients' data confidentiality was fully respected during data collection and the preparation of the manuscript.

\section{Diagnosis and Evaluations}

The International Myeloma Working Group (IMWG) criteria for the diagnosis of $\mathrm{MM}$ were adopted. The survival of all enrolled patients was followed up to December 31, 2018, or until death. Patients were categorized according to the Durie-Salmon staging system (DS) and the International Staging System (ISS). ${ }^{17,18}$ Definition of relapsed multiple myeloma based on the International Myeloma Working Group relapse criteria for multiple myeloma. ${ }^{19,20}$



Figure I Flow chart of study population.

Abbreviations: MM, multiple myeloma; PE, pleural effusion; CT, computed tomography. 
Chest CT was performed to evaluate PE. In our center, the chest CT scans were routinely examined when the patients were initially admitted, and were performed before new course of chemotherapy, or when fever, cough, expectoration, dyspnea, and other respiratory symptoms appeared, as determined by two hematologists and pulmonologists. Malignant pleural effusions (MPE), which are diagnosed based on malignant cells in the pleura or the pleural fluid.

The size of effusion was evaluated on CT scans according to the CT imaging features with anteroposterior quartile and maximum anteroposterior depth measured at the midclavicular line as described by Moy et al. The first anteroposterior-quartile effusions were small, second quartile effusions were moderate, and third and fourth quartile effusions were large. ${ }^{21}$ Evaluation of $\mathrm{PE}$ was done by radiologists and pulmonologists. For statistical considerations, data were analyzed based on the first identified PE in each patient.

\section{Statistical Analysis}

Categorical variables were described using counts and percentages, and groups were compared using a chi-square test or Fisher's exact probability test. Continuous variables were presented as means and standard deviations, and significant differences between two groups were determined with a Student's $t$-test. For non-normally distributed data, median and interquartile ranges were used to describe the features, while comparisons of the two sets were performed using a Mann-Whitney $U$-test. Survival rate was calculated using the Kaplan-Meier (KM) method. The median duration of the follow-up and its $95 \%$ CI were calculated using the reverse $\mathrm{KM}$ method. To determine the factors associated with the occurrence of pleural effusion in MM, logistic regression analysis was performed. The odds ratios (OR) with $95 \%$ confidence intervals $(\mathrm{CI})$ were presented. The statistical analysis of data was performed using SPSS 20.0 (SPSS Inc., Chicago, IL, USA) and R software (version 3.5.1) with the corresponding $\mathrm{R}$ packages. All tests were two-sided, and a value of $\mathrm{P}<0.05$ was considered statistically significant.

\section{Results}

\section{Incidence and Distribution of Pleural Effusion in Multiple Myeloma Patients}

A total of 368 patients had pleural effusion occurred during the course of treatment of MM identified on CT out of
861 patients with available CT data, yielding the incidence of $42.74 \%$. The median follow-up time of the study population was 44.9 months (95\% CI, 42.6-48.8). Characteristics of patients with and without pleural effusion are summarized in Table 1. There were no significant differences in age, sex, myeloma subtypes, and staging distribution between 861 included patients with available CT data and 46 patients without chest CT data (Supplementary Table 1).

The median time from diagnosis of multiple myeloma to pleural effusion was 6.8 months (range 0.8-33.6 months), $56.3 \%$ patients developed $\mathrm{PE}$ in the first year following the initial diagnosis of MM. PE developed in all myeloma subtypes, the distribution of myeloma types in patients with PE was similar to patients without PE. While $321(87.2 \%)$ patients developed PE presented with DS stage III, $213(57.9 \%)$ patients presented with ISS stage III. The distribution of myeloma staging using the DS and ISS was significantly different between the PEnegative and PE-positive group $(\mathrm{P}=0.015, \mathrm{P}<0.001$, respectively). The characteristics of patients developed $\mathrm{PE}$ are shown in Table 1.

The distribution features of pleural effusion in multiple myeloma patients are shown in Figure 2. 15.5\% of PEs were left-sided, $21.5 \%$ were right-sided, while both sides were affected in $63.0 \%$ of cases. In either unilateral or bilateral effusion, $82.6 \%$ of PEs were of small size, while moderate and large sizes were present in $13.0 \%$ and $4.3 \%$ of cases, respectively. Thirteen (38.2\%) malignant pleural effusions (MPE) was confirmed in 34 patients who had undergone thoracentesis, 7 (53.8\%) MPE was of small size. Pneumonia and pleural hypertrophy were frequently seen in patients with PE.

\section{Outcomes of Pleural Effusion and Overall Survival}

During the median follow-up period of 44.9 months $(95 \% \mathrm{CI}$, 42.6-48.8), the complete disappearance of PE was observed in $23(6.25 \%)$ of the patients. Decreased PE was noted in 39 $(10.6 \%)$ patients, including $\mathrm{PE}$ decreased then reoccurred in 9 patients. PE persisted in 138 (37.5\%) patients, while PE increased in $69(18.8 \%)$ patients. The response was not documented in the remaining 99 patients. In this study, 78/368 (19.8\%) patients with PE experienced dyspnea.

Thirty-four (9.24\%) patients underwent thoracentesis, $15(44.1 \%)$ patients underwent thoracentesis because of intolerable dyspnea. The median time from the initial PE 
Table I Characteristics of Patients with Multiple Myeloma

\begin{tabular}{|c|c|c|c|c|}
\hline Variables & Total Patients $(n=861)$ & PE-Positive Group $(n=368)$ & PE-Negative Group $(n=493)$ & $P$ value* \\
\hline Age, years & $59(52-66)$ & $60(54-68)$ & $58(5 I-65)$ & 0.002 \\
\hline Male, No. (\%) & $497(57.7)$ & $226(6 \mid .4)$ & $27 \mid(55.0)$ & 0.068 \\
\hline $\mathrm{CCl}$ & $3(2-4)$ & $3(2-5)$ & $3(2-4)$ & $<0.001$ \\
\hline Monoclonal protein type, n (\%) & & & & 0.957 \\
\hline $\lg G$ & $412(47.9)$ & $176(47.8)$ & $236(47.9)$ & \\
\hline $\lg A$ & $172(20.0)$ & $75(20.4)$ & 97 (19.7) & \\
\hline $\lg D$ & $58(6.7)$ & $27(7.3)$ & $31(6.3)$ & \\
\hline Light chain & $189(22.0)$ & $78(21.2)$ & III (22.5) & \\
\hline Others & $30(3.5)$ & $12(3.3)$ & $18(3.7)$ & \\
\hline DS stage, $\mathrm{n}(\%)$ & & & & 0.015 \\
\hline I & $32(3.7)$ & $12(3.3)$ & $20(4 . I)$ & \\
\hline IIA & $79(9.2)$ & $30(8.2)$ & $49(9.9)$ & \\
\hline IIB & $13(1.5)$ & $5(1.4)$ & $8(1.6)$ & \\
\hline IIIA & $575(66.8)$ & $232(63.0)$ & $343(69.6)$ & \\
\hline IIIB & $162(18.8)$ & $89(24.2)$ & $73(14.8)$ & \\
\hline ISS stage, n (\%) & & & & $<0.001$ \\
\hline I & $139(16.1)$ & $44(12.0)$ & $95(19.3)$ & \\
\hline II & $305(35.4)$ & III (30.2) & $194(39.3)$ & \\
\hline III & $4 I 7(48.4)$ & $213(57.9)$ & $204(4 I .4)$ & \\
\hline $\operatorname{Del}(17 p)$ & & & & 0.349 \\
\hline No & $54 \mid(62.8)$ & $228(62.0)$ & $313(63.5)$ & \\
\hline Yes & $60(7.0)$ & 31 (8.4) & $29(5.9)$ & \\
\hline Unknown & $260(30.2)$ & 109 (29.6) & $|5|(30.6)$ & \\
\hline Gain(|q2I) & & & & 0.911 \\
\hline No & $358(4 I .6)$ & $156(42.4)$ & $202(4 I .0)$ & \\
\hline Yes & $243(28.2)$ & $103(28.0)$ & $140(28.3)$ & \\
\hline Unknown & $260(30.1)$ & $109(29.6)$ & $|5|(30.6)$ & \\
\hline $\mathrm{t}(4 ; \mid 4)$ & & & & 0.343 \\
\hline No & $515(59.8)$ & $228(62)$ & $287(58.2)$ & \\
\hline Yes & $86(10.0)$ & 31 (8.4) & 55 (II.2) & \\
\hline Unknown & $260(30.2)$ & 109 (29.6) & I5I (30.6) & \\
\hline$t(14 ; 16)$ & & & & 0.929 \\
\hline No & $579(67.2)$ & $250(67.9)$ & $329(66.7)$ & \\
\hline Yes & $22(2.6)$ & $9(2.4)$ & $13(2.6)$ & \\
\hline Unknown & $260(30.2)$ & $109(29.6)$ & $|5|$ (30.6) & \\
\hline $\mathrm{t}(1 \mathrm{I} ; 14)$ & & & & 0.929 \\
\hline No & $513(59.6)$ & $222(60.3)$ & 291 (59.0) & \\
\hline Yes & $88(10.2)$ & $37(10.1)$ & $51(10.3)$ & \\
\hline Unknown & $260(30.2)$ & 109 (29.6) & $|5|$ (30.6) & \\
\hline
\end{tabular}

Notes: Data are presented as median (interquartile range) or \%. *For comparisons between PE-negative group and PE-positive group. Abbreviations: PE, pleural effusion; CCI, Charlson Comorbidity Index; DS, Durie-Salmon; ISS, International Scoring System.

was found to the first thoracentesis was 1.0 months (range, 0-7.5 months).

One hundred and eighty-four (32.1\%) patients died during the follow-up duration. The median overall survival
(OS) was 82.4 months (95\% CI, 72.5-89.6), and the 5-year OS rates were $62.0 \%$ (95\% CI, 56.9\%-67.5\%). KaplanMeier (KM) curves of overall survival in MM patients following the initial diagnosis of $\mathrm{MM}$ are showed in 
A



C

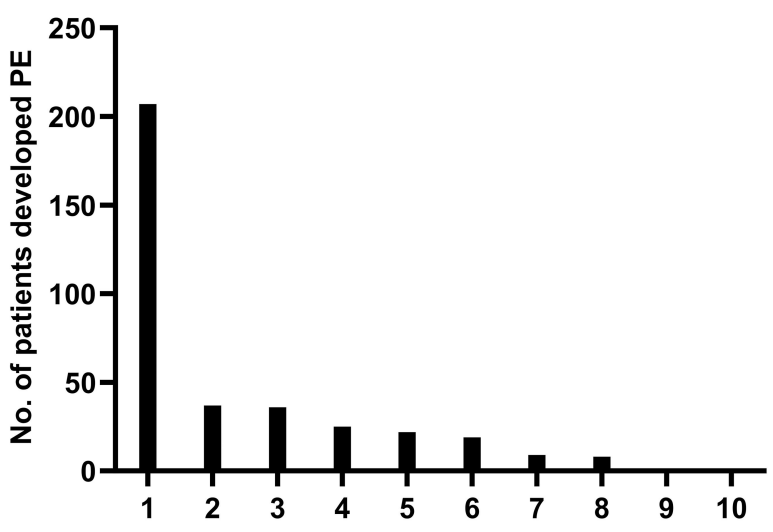

Time following the initial diagnosis of MM (years)
B

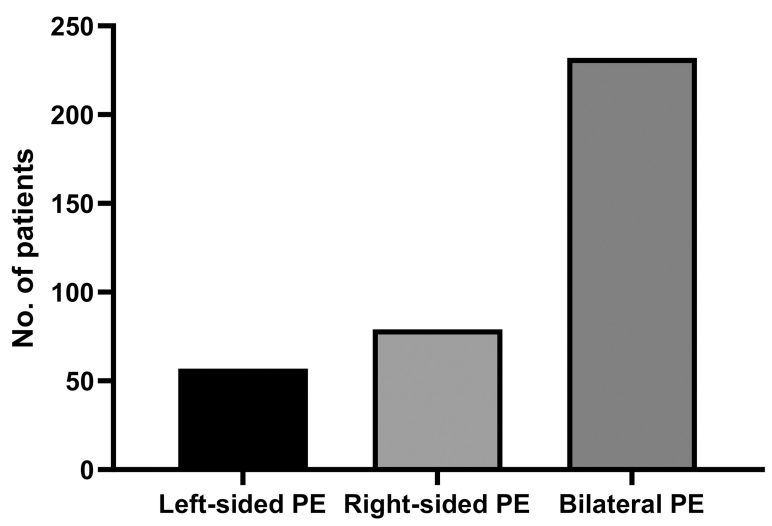

D

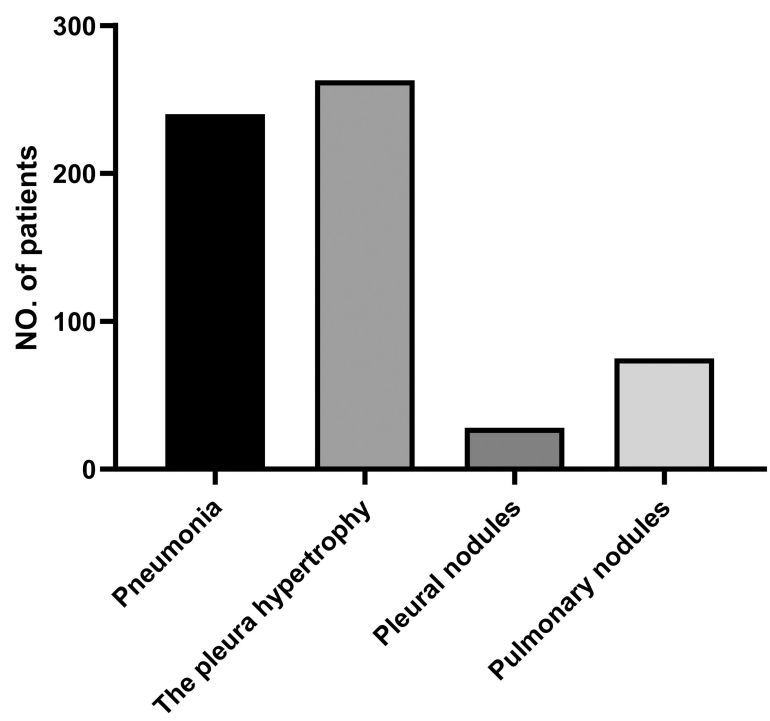

Figure 2 The distribution features of pleural effusion in multiple myeloma patients $(n=368)$. (A) The effusion size distributions; (B) location of PE; (C) the occurrence time of PE; (D) other pulmonary CT findings.

Abbreviations: MM, multiple myeloma; PE, pleural effusion; $\mathrm{CT}$, computed tomography.

Figure 3. Patients with PE showed significantly worse survival since the initial diagnosis of MM than those without PE (unadjusted HR, 2.249; 95\% CI, 1.774-2.852). No difference in survival was found between patients with small PE and those with moderate to large PE (unadjusted HR, 1.402; 95\% CI, 1.037-1.896).

\section{Risk Factors for Pleural Effusion in Multiple Myeloma Patients}

We explored the risk factors for pleural effusion using logistic regression, as shown in Table 2. Our study investigated patients who presented pleural effusion at the time of initial MM diagnosis or at the time of relapse. Fifty-two (9.8\%) presented pleural effusion at the time of initial MM diagnosis among 528 patients who were newly diagnosed in our center. Among the 208 patients who were relapsed, $143(68.8 \%)$ presented pleural effusion at the time of relapse. Input variables for logistic regression analysis were selected from significant variables obtained from the univariate analysis and variables related to the occurrence of $\mathrm{PE}$ that were reported in the previous literature, the final model contains 13 variables.

Clonal plasma cell proportion in the bone marrow at the time of initial MM diagnosis (OR, 1.373; 95\% CI, 1.153-1.634; $\mathrm{P}=0.009)$ and amyloidosis (OR, 1.791; 95\% CI, 1.408-2.279; $\mathrm{P}=0.024$ ) were independent risk factors for the occurrence of pleural effusion at the initial diagnosis of MM. Clonal plasma cell proportion in the bone marrow at the time of MM relapse (OR, 1.853; 95\% CI, 1.451-2.368; $\mathrm{P}=0.038$ ), pneumonia (OR, 1.309; 95\% CI, 1.143-1.498; $\mathrm{P}=0.008$ ) and heart failure 
A $\quad$ Pleural Effusion + without $\mathrm{PE}+$ with $\mathrm{PE}$

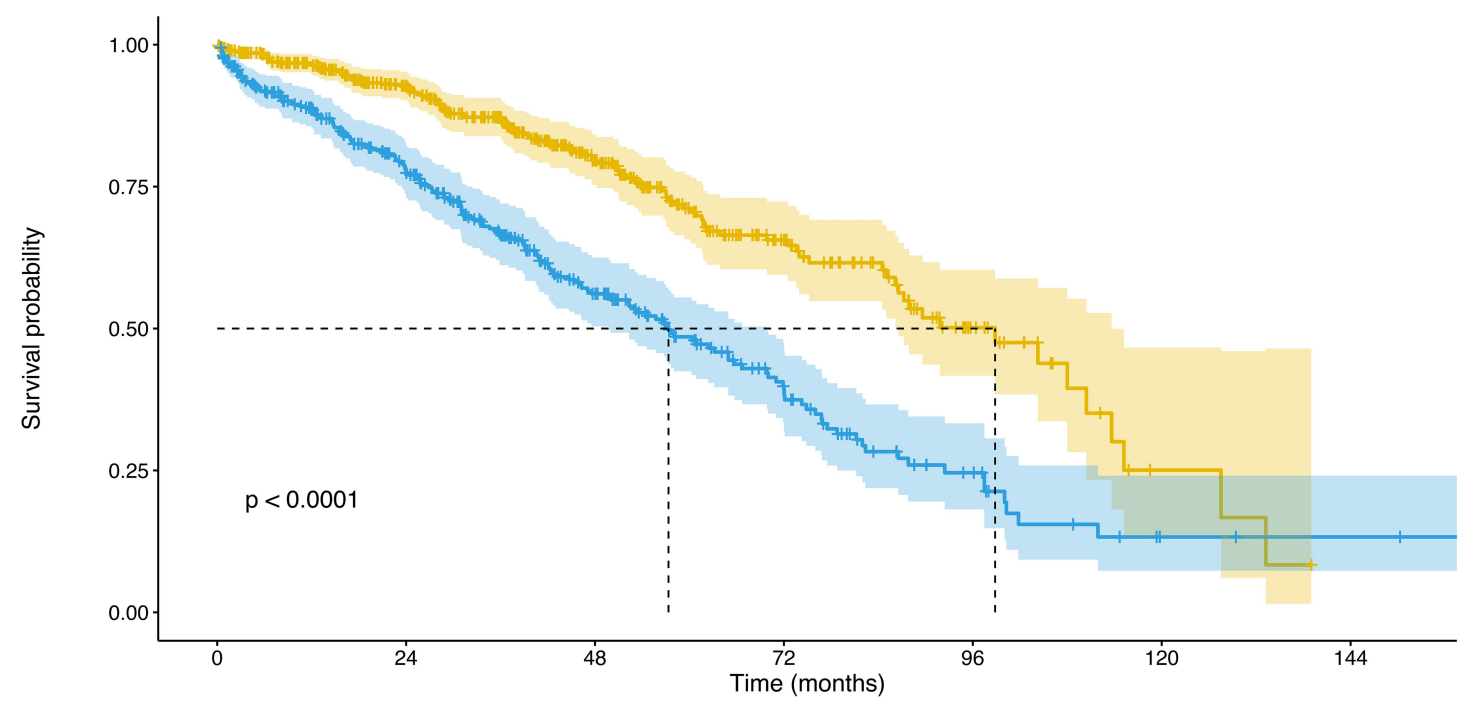

Number at risk

$\begin{array}{cccccccc}\text { without PE } & 493 & 333 & 170 & 70 & 22 & 3 & 0 \\ \text { with PE } & 368 & 226 & 111 & 50 & 17 & 3 & 2\end{array}$

B

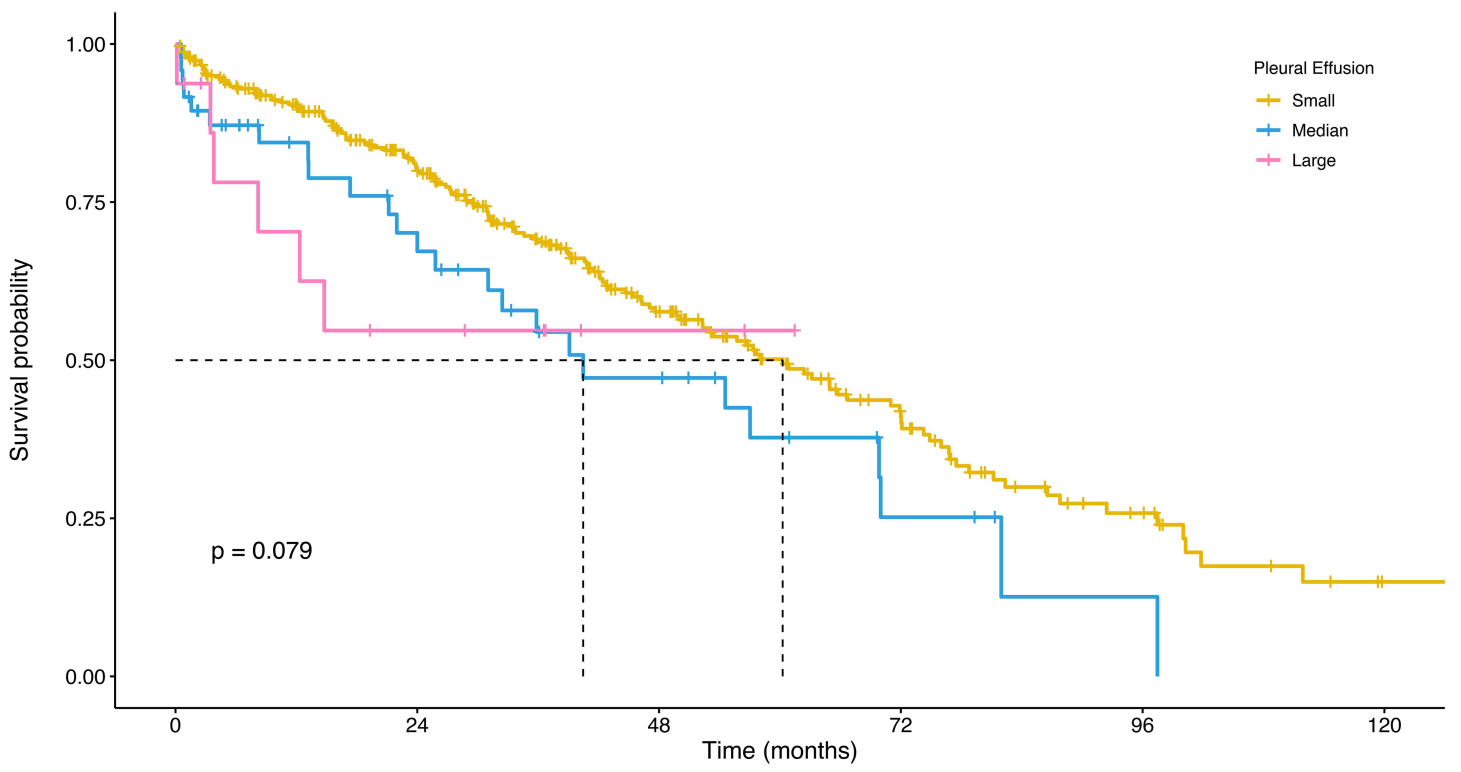

Number at risk

\begin{tabular}{|c|c|c|c|c|c|}
\hline Small & 304 & 196 & 96 & 46 & 16 \\
\hline Median & 48 & 24 & 13 & 4 & 1 \\
\hline Large & 16 & 6 & 2 & 0 & 0 \\
\hline
\end{tabular}

Figure 3 Kaplan-Meier (KM) curves of overall survival in MM patients following the initial diagnosis of MM. (A) Curves between patients with PE and patients without PE; (B) curves between patients with small PE and those with moderate to large PE.

Abbreviations: MM, multiple myeloma; PE, pleural effusion. 
Table 2 Risk Factors for the Occurrence of Pleural Effusion in MM Patients*

\begin{tabular}{|l|c|c|}
\hline Characteristics & OR (95\% Cl) & P value \\
\hline Newly diagnosed patients & & 0.009 \\
Plasma cell proportion (>38.5\% vs $\leq 38.5 \%)$ & $1.373(1.153-1.634)$ & 0.024 \\
Amyloidosis & $1.791(1.408-2.279)$ & 0.038 \\
\hline Patients with relapse & & 0.008 \\
Plasma cell proportion (>38.5\% vs $\leq 38.5 \%)$ & $1.853(1.451-2.368)$ & 0.031 \\
Pneumonia & $1.309(1.143-1.498)$ & \\
Presence of heart failure & $1.815(1.387-2.374)$ & \\
\hline
\end{tabular}

Notes: *Variables in the logistic regression that did not have a significant independent association with the occurrence of pleural effusion in multiple myeloma patients: Age, Male, M-protein types, the International Staging System (ISS) stage, Durie-Salmon staging system (DS), Fluorescence in situ hybridization (FISH) test: del(I7), gain(I 2 I), $t$ $(4 ; 14), t(14 ; 16)$ and $t(I I ; 14)$, Presence of renal failure, History of tuberculous, Hypoproteinemia.

Abbreviations: MM, multiple myeloma; OR, odds ratio; $\mathrm{Cl}$, confidence interval.

(OR, 1.815; 95\% CI, 1.387-2.374; $\mathrm{P}=0.031)$ were independent risk factors for the occurrence of pleural effusion at relapse of MM.

\section{Discussion}

To the best of our knowledge, this was the largest series dealing with $\mathrm{PE}$ in patients with MM. The noteworthy finding in this study was the incidence of $\mathrm{PE}$ in $\mathrm{MM}$ patients. Namely, the incidence of PE in MM patients was $42.7 \%$, which is much higher than the data from prior reports. Lower incidence in other studies may reflect the fact that most previous studies were in the form of case reports, and only two studies mentioned the frequency of $\mathrm{PE}$ in the range between $10.7 \%$ and $13.9 \% ;^{15,16}$ however, there were no detailed evaluations. Moreover, a higher incidence of PE in our series may be due to the meticulous attention paid to detecting PE in our hospital. Beijing Chao-Yang Hospital not only has the Beijing Institute of Respiratory Diseases, well recognized at the national level, but also houses one of the largest myeloma treatment centers in China. Our study suggested that the incidence of PE in MM patients is actually notable, and it is likely that $\mathrm{MM}$ is often overlooked in patients with $\mathrm{PE}$ in other centers.

We noted that patients with PE showed worse survival than those without PE. The statistical calculation is hinging on the presence or absence of pleural effusion, whatever the clearly etiologies of PEs. It is just the presence of $\mathrm{PE}$ has an impact on prognosis, which is why it is very important to identify it. Pleural effusion is almost always a manifestation of one or more underlying primary conditions. It is possible that presence of effusion is a marker of poor prognosis by virtue of the severe tumor burden or worse host comorbidity status.
Hitherto, the distribution of the PE in MM patients has not been studied. Previous studies reported inconsistent and unclear data on incidence of PE among different subtypes of MM. A higher prevalence of $\operatorname{IgG}$ and $\operatorname{IgA}$ subtypes was reported in previous case reports. ${ }^{2-25}$ Here we found that PE occurred in all classes of myeloma subtypes. We noted that majority of PEs in our study were of small size. It is noteworthy that even small PE, its presence is an important prognostic factor of worse survival. No difference in survival was found between patients with small $\mathrm{PE}$ and those with moderate to large PE. In addition, MPE is not always massive, given that we found that in more than 50\% MPEs was confirmed in 34 patients who had undergone thoracentesis in our study were of small size. Small PE might represent an early phase of malignant PE or severe comorbid disease. We suggested that more attention should be paid to pleural effusion, even small amounts of pleural effusion.

Another important aspect of our study was related to identification of risk factors associated with the occurrence of PE. Through logistic regression analysis, we demonstrated that plasma cell proportion was an independent risk factor for the occurrence of PE either at the initial diagnosis of $\mathrm{MM}$ or at the time of MM relapse. This association remained significant after adjustment for age, sex, M-protein types, the ISS or DS stage, fluorescence in situ hybridization (FISH) test, presence of heart disease or renal failure, history of tuberculous and hypoproteinemia. MM is a malignancy of the B-cell lineage, characterized by the accumulation of clonal plasma cells in the bone marrow, leading to excessive production of immunoglobulins. High proportion of clonal plasma cells in the bone marrow indicates greater severity of the disease..$^{26,27}$ It is supposed that a factor of the development of PE in MM 
patient is the production of large quantities of immunoglobulins, which leads to high colloid osmotic pressure of the fluid. $^{28}$

Additionally, in this study, amyloidosis was found to be an independent risk factor for the occurrence of PE at the initial diagnosis of MM. In amyloidosis, amyloid protein is derived from immunoglobulin light chains, and most often involves the kidneys and the heart. Direct pleural amyloidosis can also lead to pleural effusions. Sunny et al reported a case of exudate and amyloidosis in thoracoscopic pleural biopsy. ${ }^{29}$ Pneumonia and heart failure were found to be significant independent predictors for the occurrence of pleural effusion at relapse by logistic regression. In our study, more than $65 \%$ of patients experienced pneumonia at the onset of pleural effusion in our study. For these MM patients undergoing chemotherapy, infection does play a role in the development of PE. In addition, many of MM patients have heart failure. The capillary hydrostatic pressure might be increased lead to the occurrence of PE. Multiple myeloma can have direct and indirect detrimental effects on cardiac function. Several drug classes used in the treatment of $\mathrm{MM}$ are known to increase the risk of cardiac events, such as doxorubicin, anthracycline, lenalidomide, pomalidomide, carfilzomib and bortezomib. ${ }^{30}$

Since the incidence of PE was higher than expected, these patients with PE actually pose a diagnostic and therapeutic challenge in need of better management approaches. Infection and heart failure are important factors leading to PE. ${ }^{16,31}$ Also, considering the high prevalence of tuberculosis in China, tuberculosis might have been overlooked in these MM patients with PE. These are possibly treatable causes. On the other hand, in patients underwent thoracentesis, MPE was confirmed in 13/34 (38.2\%) patients. Previous literature reported the MPE in MM patients frequency of $0.8 \%$ to $2.65 \% .^{32-34}$ A part of the effusions in 334 patients who did not receive additional evaluation to determine the cause of PE may be MPE, but they were not identified. Complete disappearance of PE was observed in only $6.5 \%$ patients, and most PE persisted or increased in the remaining patients. If PE went undetected or did not be pay attention to, it may result in some patients end up receiving suboptimal treatment. Identify reversible causes of PE or MPE conducive to clinical decision making. In addition, recent evidence suggests that indwelling pleural catheters are safe in hematologic malignancies. ${ }^{35}$ Therefore, a diagnostic thoracentesis or further evaluation of $\mathrm{PE}$ should be performed widely in MM patients.

Though our investigation provided first comprehensive study to evaluate epidemiology, clinical characteristics, risk factors, and prognosis of PE in MM patients, it had a few limitations. First of all, our study was a real-world, single-center, retrospective cohort study, which resulted in incomplete data and inability to control examinations and treatment. Some patients were asymptomatic and may not have been picked up in the absence of routine evaluation. Long follow-up is often associated with missing data, and is likely to have bias errors. Second, few patients underwent thoracentesis, which might have limited the interpretative power on the cause of the effusion. Finally, future research will be required to determine if $\mathrm{PE}$ is a sign for therapy change and if more aggressive therapy after the diagnosis of PE can improve prognosis.

\section{Conclusions}

The incidence of PE in MM patients is notable and PE can occur in all MM subtypes. PE indicates a poor prognosis, even small amounts of effusion. PE is a problem worthy of attention, especially in patients with high plasma cell proportion, amyloidosis or complicated with pneumonia and heart failure.

\section{Acknowledgments}

The authors thank the patients, their families, and all investigators who participated in the study.

\section{Disclosure}

The authors report no conflicts of interest in this work.

\section{References}

1. Light RW. Pleural effusions. Med Clin North Am. 2011;95 (6): 1055-1070.

2. Light RW. Clinical practice. Pleural effusion. $N$ Engl J Med. 2002;346 (25):1971-1977.

3. Sahn SA, Heffner JE. Pleural fluid analysis. In: Light RW, Lee YCG, editors. Textbook of Pleural Diseases. 2nd. London, UK: Arnold Press; 2008:209-216

4. BTS Pleural Guideline Group. Investigation of a unilateral pleural effusion in adults: British Thoracic Society pleural disease guideline 2010. Thorax. 2010;65(Suppl 2):ii4-ii17.

5. Goldstraw P, Crowley J, Chansky K, et al. The IASLC lung cancer staging project: proposals for the revision of the TNM stage groupings in the forthcoming (seventh) edition of the TNM Classification of malignant tumours. $J$ Thorac Oncol. 2007;2(8):706-714.

6. Ryu JS, Azra M, Lee SK, et al. Prognostic impact of minimal pleural effusion in non-small-cell lung cancer. J Clin Oncol. 2014;32 (9):960-967. 
7. Nakaya A, Fujita S, Satake A, et al. Clinical significance of dasatinib-induced pleural effusion in patients with de novo chronic myeloid leukemia. Hematol Rep. 2018;10(3):7474.

8. Porkka K, Khoury HJ, Paquette RL, et al. Dasatinib $100 \mathrm{mg}$ once daily minimizes the occurrence of pleural effusion in patients with chronic myeloid leukemia in chronic phase and efficacy is unaffected in patients who develop pleural effusion. Cancer. 2010;116 (2):377-386.

9. Röllig C, Knop S, Bornhäuser M, et al. Multiple myeloma. Lancet. 2015;385(9983):2197-2208.

10. Siegel RL, Miller KD, Jemal A, et al. Cancer statistics, 2016. $C A$ Cancer J Clin. 2016;66(1):7-30.

11. Chen JH, Chung $\mathrm{CH}$, Wang $\mathrm{YC}$, et al. Prevalence and mortality-related factors of multiple myeloma in Taiwan. PLoS One. 2016;11(12):e0167227.

12. Park HJ, Park EH, Jung KW, et al. Statistics of hematologic malignancies in Korea: incidence, prevalence and survival rates from 1999 to 2008. Korean J Hematol. 2012;47(1):28-38.

13. Lu J, Lu J, Chen W, et al. The Chinese medical doctor association hematology branch. Clinical features and treatment outcome in newly diagnosed Chinese patients with multiple myeloma: results of a multicenter analysis. Blood Cancer J. 2014;4:e239. doi:10.1038/ bcj. 2014.55

14. Kyle RA, Rajkumar SV. Multiple myeloma. Blood. 2008;111:2962-2972.

15. Wang Z, Xia G, Lan L, et al. Pleural effusion in multiple myeloma. Intern Med. 2016;55(4):339-345.

16. Byun JM, Kim KH, Choi IS, et al. Pleural effusion in multiple myeloma: characteristics and practice patterns. Acta Haematol. 2017;138(2):69-76.

17. Rajkumar SV. Multiple myeloma: 2016 update on diagnosis, risk-stratification, and management. Am J Hematol. 2016;91 (7):719-734.

18. Rajkumar SV. Myeloma today: disease definitions and treatment advances. Am J Hematol. 2016;91(1):90-100.

19. Kyle RA, Rajkumar SV. Criteria for diagnosis, staging, risk stratification and response assessment of multiple myeloma. Leukemia. 2009;23:3-9.

20. Rajkumar SV, Harousseau JL, Durie B, et al. Consensus recommendations for the uniform reporting of clinical trials: report of the International myeloma workshop consensus panel 1. Blood. 2011;117:4691-4695.
21. Moy MP, Levsky JM, Berko NS, et al. A new, simple method for estimating pleural effusion size on CT scans. Chest. 2013;143 (4):1054-1059.

22. Juan NR. Pleural effusion in multiple myeloma. Chest. 1994;105:622-624.

23. Kamble R, Wilson CS, Fassas A, et al. Malignant pleural effusion of multiple myeloma: prognostic factors and outcome. Leuk Lymphoma. 2005;46(8):1137-1142.

24. Zhang LL, Li YY, Hu CP, et al. Myelomatous pleural effusion as an initial sign of multiple myeloma - a case report and review of literature. J Thorac Dis. 2014;6(7):E152-E159.

25. Saidane O, Slouma M, Haouet S, et al. Cutaneous and pleural involvement in a patient with multiple myeloma. BMJ Case Rep. 2015;201.

26. Rajkumar SV. Multiple myeloma: 2018 update on diagnosis, risk-stratification, and management. Am J Hematol. 2018;93 (8):981-1114.

27. Gerecke C, Fuhrmann S, Strifler S, et al. The diagnosis and treatment of multiple myeloma. Dtsch Arztebl Int. 2016;113(27-28):470-476.

28. Chetan BP, Neeraj G, Rakesh CG, et al. Varna Indushekar Myelomatous pleural effusion-thoracoscopic evaluation of a rare entity. Lung India. 2015;32(5):505-507.

29. George S, Ravindran M, Anandan PT, et al. Primary systemic amyloidosis: a rare cause for pleural effusion. Respir Med Case Rep. 2014;13:39-42.

30. Kistler K, Rajangam K, Faich G. Cardiac event rates in patients with newly diagnosed and relapsed multiple myeloma in US clinical practice. Blood. 2012;120:2916.

31. Dipenkumar M, Hyejeong J, Seongho K, et al. Incidence, etiology, and outcome of pleural effusions in allogeneic hematopoietic stem cell transplantation. Am J Hematol. 2016;91(9):E341-E347.

32. Kim YJ, Kim SJ, Min K, et al. Multiple myeloma with myelomatous pleural effusion: a case report and review of the literature. Acta Haematol. 2008;120(2):108-111.

33. Arora P, Gupta SK, Mallik N, et al. Flow cytometry in diagnosis of myelomatous pleural effusion: a case report. Indian J Hematol Blood Transfus. 2016;32(Suppl 1):138-342.

34. Mangla A, Agarwal N, Kim GJ, et al. Primary malignant myelomatous pleural effusion. Clin Case Rep. 2016;4(8):803-806.

35. Vakil E, Jimenez CA, Faiz SA, et al. Pleural effusions in hematologic malignancies and their management with indwelling pleural catheters. Curr Opin Pulm Med. 2018;24(4):384-391.
International Journal of General Medicine

\section{Publish your work in this journal}

The International Journal of General Medicine is an international, peer-reviewed open-access journal that focuses on general and internal medicine, pathogenesis, epidemiology, diagnosis, monitoring and treatment protocols. The journal is characterized by the rapid reporting of reviews, original research and clinical studies across all disease areas. The manuscript management system is completely online and includes a very quick and fair peer-review system, which is all easy to use. Visit http://www.dovepress.com/ testimonials.php to read real quotes from published authors. 